\title{
Hacia una nueva generación de reformas para mejorar la salud de las poblaciones
}

\author{
Comité Editorial: Daniel López-Acuña, Pedro Brito, Pedro Crocco, \\ Alberto Infante, José María Marín y José Luis Zeballos
}

\section{¿QUÉ HAN SIDO HASTA AHORA LAS REFORMAS DEL SECTOR DE LA SALUD EN LAS AMÉRICAS?}

En los últimos veinte años se han producido, no solamente en las Américas sino prácticamente en todo el mundo, numerosos esfuerzos dirigidos a introducir cambios en la organización y el financiamiento de los sistemas de salud, así como en el arreglo institucional propio del sector. Estos cambios, conocidos como reformas del sector de la salud, han sido sumamente diversos y, por lo general, forman parte de procesos más amplios de transformación de la vida económica, social y política de los países y de modernización del Estado.

En la I Cumbre de las Américas (Miami, 1994), los Jefes de Estado y de Gobierno de los países de las Américas se pronunciaron con toda claridad sobre la necesidad de que las reformas del sector de la salud estuvieran orientadas a garantizar un acceso equitativo a servicios básicos de salud. No se pretende reformar por reformar, sino reformar con un sentido y una dirección que supongan ofrecer oportunidades a los excluidos, cubrir a los más necesitados y corregir las fallas de equidad que hoy existen en materia de situación de salud, de acceso a los servicios y de financiamiento del sector.

En el Plan de Acción de la I Cumbre de las Américas se incluyó la convocatoria de una reunión continental especial para discutir los avances y los desafíos que enfrentaban los procesos de reforma del sector de la salud en las Américas. En dicho foro, realizado en septiembre de 1995 en el marco del Consejo Directivo de la Organización Panamericana de la Salud (OPS), se produjeron debates sobre el estado de las reformas que llevaron a los Gobiernos Miembros de la Organización a definir cinco "principios rectores" de las reformas del sector de la salud que deberían guiar la marcha de las transformaciones y servir de base para su seguimiento y evaluación. Dichos principios son: equidad, eficiencia, efectividad y calidad, sostenibilidad y participación social.
Aunque en muchas reformas emprendidas en la Región se pusieron de relieve solamente algunos de estos principios, ha habido consenso en el sentido de que las reformas no deberían oponerse a estos criterios y de que la "reforma ideal" sería aquella en la cual los cinco aspectos hubieran mejorado al final del proceso. Esta búsqueda de consenso en torno a los principios rectores ha sido útil no solo para dar seguimiento a las reformas, sino también para debatir cómo reorientarlas cuando se hacen patentes sus limitaciones e insuficiencias.

En la misma Cumbre de Miami, los Jefes de Estado y de Gobierno emitieron el mandato, conferido a la OPS, de poner en marcha un proceso de seguimiento y evaluación de las reformas del sector de la salud en los países de las Américas, para lo cual, con el apoyo de los países miembros y con la colaboración de la Agencia de Estados Unidos para el Desarrollo Internacional, se ha puesto en marcha la "Iniciativa Regional de Reforma del Sector de la Salud en América Latina y el Caribe", que está permitiendo examinar el curso que han seguido las reformas y los ajustes y redireccionamientos requeridos para que realmente impacten en objetivos coherentes con los principios rectores mencionados.

Los resultados del seguimiento y evaluación de las reformas del sector de la salud en las Américas, llevado a cabo hasta ahora por la OPS en respuesta a dicho mandato, permiten realizar una apreciación preliminar del efecto de las reformas que puede resumirse como sigue:

- Equidad. Solo algunas de las reformas parecen estar contribuyendo, lentamente, a la reducción de las disparidades en la cobertura de algunos servicios y programas básicos, aunque subsisten dificultades para medir esta dimensión de los cambios del sector. En la mayoría de los países, las reformas no están contribuyendo a la reducción de las disparidades injustificadas en la distribución de los recursos y servicios, y es sumamente difícil valorar en qué medida han con- 
tribuido a reducir las disparidades en la situación de salud.

- Efectividad y calidad. Se ha avanzado relativamente poco en la mejora de la efectividad global del sistema y en el cumplimiento de los objetivos de calidad de la atención y satisfacción de los usuarios.

- Eficiencia. Según los análisis realizados, se ha avanzado más en la productividad y el desarrollo de métodos de adquisición de insumos que en la reorientación de la asignación de recursos. Por ejemplo, no ha habido cambios importantes en la asignación de recursos para abordar problemas con grandes externalidades o para aumentar la protección social en el campo de la salud.

- Sostenibilidad. Se está tratando de ceñir los gastos a los ingresos del sistema, pero muy pocos países están aumentando la generación de recursos a mediano o largo plazo para ampliar o mantener el nivel actual de prestación de servicios. Esta situación se agrava con la gran dependencia del financiamiento externo observada en muchos países y la falta de mecanismos para sustituir estas corrientes de recursos cuando cesan. Además, la cuestión de la legitimidad social apenas ha sido abordada.

- Participación social. Hay indicios de una mayor receptividad de los gobiernos como resultado de los planes de reforma del sector de la salud. Esto introduce importantes contrapesos en el sistema, pero todavía queda por ver si ello influirá en la reorientación del curso de las reformas.

Una carencia adicional de las reformas ha sido que, hasta ahora, los problemas, las políticas y las intervenciones referidas a los recursos humanos han tenido un lugar poco destacado en la agenda $y$, en el mejor de los casos, solamente han merecido un manejo instrumental como parte de otras intervenciones orientadas a la eficiencia y a la productividad. Paradójicamente, esto ha sucedido al mismo tiempo que las transformaciones del Estado, de la economía y de las instituciones determinaban una revolución en las condiciones económicas del personal y en las relaciones de trabajo hasta entonces vigentes, es decir, cuando más se necesitaba fortalecer la función estatal e institucional de desarrollo de los recursos humanos. Otra dimensión esencial de los recursos humanos en las reformas es la política. El personal de salud, individual y colectivamente, es un protagonista de los procesos de cambio del sector y su participación, a favor o en contra de esos procesos, suele determinar la viabilidad y la sostenibilidad de los cambios.

En resumen, las motivaciones que han impulsado las reformas se han centrado hasta ahora en algunos aspectos de gestión y en ciertos aspectos relacionados con la eficiencia económica. En la práctica, las cuestiones de equidad, protección social en salud y salud pública han quedado relegadas a un plano secundario. La calidad de la atención y, más concretamente, el modelo de atención han sido temas marginales en los debates sobre las reformas en la mayoría de los países. Las reformas tampoco han convergido adecuadamente con los procesos de cambio de la seguridad social en salud que se han ido dando en paralelo, lo cual ha dificultado a menudo alcanzar a los grupos de la población más desfavorecidos.

\section{EL CONTEXTO AL QUE SE ENFRENTAN LAS REFORMAS DE LA SALUD EN LAS AMÉRICAS EN EL UMBRAL DEL SIGLO XXI}

América Latina y el Caribe viven una situación de alta inestabilidad económica y social, de resquebrajamiento de la gobernabilidad y de crecimiento asombroso de la pobreza y de la inequidad. Más que nunca, esto hace necesario que los cambios que se introduzcan en los sectores sociales, como el de la salud, contribuyan a la construcción de sociedades incluyentes de todos sus ciudadanos y no a la profundización de la exclusión, de la marginación y de la desprotección social. Por tanto, si se quieren crear procesos orientados con criterios de equidad y sustentados en el valor de la solidaridad como forma de alcanzar verdaderamente a los excluidos de nuestras sociedades y fomentar la cohesión social, es fundamental que las reformas del sector de la salud se planteen hasta qué punto están realmente mejorando los indicadores de salud y satisfaciendo las necesidades de los más desprotegidos.

En el umbral del nuevo milenio, los países de América Latina y el Caribe enfrentan un enorme desafío de proporciones crecientes: garantizar a todos sus ciudadanos un grado básico de protección social en materia de salud que contribuya a eliminar las desigualdades en el acceso a servicios básicos de calidad para todas las personas y que proporcione a los grupos sociales hasta ahora excluidos la oportunidad de recibir atención integral con miras a la satisfacción de sus necesidades y demandas de salud, sin que su capacidad de pago sea un factor restrictivo.

La Región muestra hoy un crecimiento poblacional que se combina con el estancamiento del crecimiento económico, el aumento del desempleo, el crecimiento de la economía informal, el recrudecimiento de la pobreza en términos absolutos y relativos y la profundización de las disparidades en 
la distribución de los ingresos. Todo ello lleva a que el problema de la exclusión económica, social, étnica y cultural adquiera proporciones cada vez más serias y a que los mecanismos existentes de protección social en salud, diseñados en su día para garantizar a la población una serie de prestaciones en virtud de medidas de carácter público, resulten hoy insuficientes para dar respuesta a los nuevos problemas que se presentan en este ámbito.

\section{HACIA UNA NUEVA GENERACIÓN DE REFORMAS DEL SECTOR CENTRADAS EN LA SALUD DE LAS POBLACIONES}

Las reformas del sector de la salud se han centrado principalmente en cambios financieros, estructurales e institucionales de los sistemas de salud y en reajustes de la organización y la administración de la atención a la salud de las personas. Mucha menos atención han recibido las mejoras del desempeño del sistema centradas en la reducción de las inequidades en las condiciones de salud y en el acceso a la atención de salud y su financiamiento; en la reducción de la desprotección social en salud; en el aumento de la eficacia de las intervenciones en salud; en la promoción de la calidad de la atención; en el fortalecimiento de la función rectora de las autoridades de salud, y en la mejora en la práctica de la salud pública.

Estas reformas se realizan en un momento en que el problema de la exclusión económica, social y cultural está empeorando. Las medidas actuales de protección social en el ámbito de la salud, que consisten en sistemas de asistencia social y seguridad social, no bastan para hacer frente a los actuales y a los nuevos problemas. Por consiguiente, la tarea fundamental de los países consiste en ofrecer a los ciudadanos, independientemente de su capacidad de pago, protección social básica universal en materia de salud que lleve a una reducción de la desigualdad en el acceso a servicios necesarios, eficaces y de buena calidad. Ello contribuirá simultáneamente a fortalecer el desarrollo y la cohesión social, tal como se viene insistiendo recientemente desde distintos organismos internacionales.

Hay un espacio simultáneo de necesidad y de oportunidad que se abre en las agendas de cambio del sector para poder avanzar en la construcción del acceso equitativo a la atención de la salud. Pero para eso es necesario consolidar la cobertura efectiva de los grupos excluidos, particularmente de los pertenecientes al sector informal de la economía y a los grupos marginados por razones culturales o geográficas.

En consecuencia, se presenta una gran oportunidad para considerar en la nueva generación de reformas del sector el diseño, la ejecución y la evaluación de mecanismos innovadores de ampliación de la cobertura de la seguridad social de salud, dirigidos hacia los grupos que no están insertos en el sector formal de la economía ni tienen capacidad contributiva para afiliarse a los regímenes habituales de la seguridad social de salud.

Se hace necesario apelar a nuevas fórmulas que se apoyen más en el capital social de los grupos excluidos, que se dirijan a racionalizar los mecanismos de financiamiento y la regresividad del gasto de bolsillo en la salud, que hoy supone una mayor carga financiera para los hogares y para las personas más desfavorecidas, y que aprovechen los mecanismos comunitarios de organización cooperativa para encontrar respuestas complementarias a la protección social de la salud que hoy se ofrece mediante los esfuerzos de la asistencia social y de la seguridad social, los cuales desafortunadamente no llegan a todos los ciudadanos.

En vista de lo anterior, la Organización Internacional del Trabajo y la OPS han suscrito un memorándum de entendimiento para impulsar una iniciativa regional de apoyo a los esfuerzos de los países miembros en materia de extensión de la protección social de salud a los grupos excluidos.

Sin embargo, las innovaciones en materia de protección social en el campo de la salud por sí solas no bastan. Deben ir acompañadas por una reorientación de los sistemas y los servicios de salud con criterios de promoción de la salud y de prevención de la enfermedad. Los pobres no solo tienden a recibir servicios curativos de calidad inferior, sino que también son precisamente el grupo que más necesita servicios reorientados con criterios de promoción de la salud. Por lo tanto, sin una transformación del modelo de atención, seguirá habiendo una gran falta de equidad en la integralidad y efectividad de los servicios de salud.

Todo esfuerzo importante para inducir cambios en el sistema de salud debería fortalecer las funciones esenciales de la salud pública que están a cargo de las autoridades de salud de cada país, como parte de los esfuerzos generales para redefinir y fortalecer la función rectora de las autoridades sanitarias, las infraestructuras y las prácticas sociales en salud pública. En muchos países se está trabajando en la explicitación de las funciones esenciales de la salud pública y en la creación y aplicación de instrumentos para medir el desempeño de dichas funciones. También se trata de que estos elementos se usen para mejorar la práctica de la salud pública y para rehabilitar la infraestructura de los servicios de salud pública.

La relación entre las funciones esenciales de la salud pública y la reorientación de los servicios de salud con criterios de promoción de la salud 
opera en dos niveles. En primer término, el fortalecimiento de la capacidad del Estado para desempeñar cada una de las funciones esenciales de la salud pública facilita la consecución de los objetivos de la promoción de la salud. Un desempeño adecuado en todos los campos de la salud pública da mayor legitimidad a las autoridades de salud como componentes responsables, capaces y críticos del sistema de salud, y aumenta la capacidad del sistema para cumplir la función de promoción de la salud. En segundo lugar, con la formulación de funciones esenciales de la salud pública y la evaluación de su desempeño se refuerzan las alianzas intersectoriales, que son indispensables para la promoción de la salud. Esta coyuntura histórica a la que se enfrentan las reformas del sector de la salud al iniciarse el siglo XXI podría ofrecer una oportunidad importante para superar las insuficiencias de las reformas actuales que han omitido la salud pública como responsabilidad social.

La reorientación de los sistemas y servicios ha de hacerse también con criterios de calidad en el sentido de centrarlos en la solución de problemas y en el impulso a una mayor integralidad, a una mayor orientación hacia lo público, a una mayor continuidad de los cuidados, fortaleciendo y coordinando mucho mejor las diferentes redes de prestadores en un territorio determinado, $\mathrm{y}$ a una mejor capacidad de respuesta a las características individuales y colectivas y a las demandas de sus usuarios efectivos y potenciales. Los sistemas y servicios deberían situar al paciente adecuadamente atendido y satisfecho en el centro de su quehacer y ser sentidos como accesibles y próximos por la población, lo cual está vinculado también con la capacidad de las autoridades de salud para desempeñar cabalmente su papel rector del sector y para ejercer las funciones esenciales de la salud pública que les son propias e indelegables. No cabe duda que ello aumentaría su legitimidad social y contribuiría a la sostenibilidad de sus acciones.

En una perspectiva nueva y con otros objetivos de cambio del sector, los recursos humanos deberán ser parte esencial de las agendas de reforma. En este sentido, deberán considerarse los siguientes aspectos centrales:

- La formulación, viabilidad y ejecución de políticas de desarrollo de recursos humanos orientadas tanto a sustentar los cambios del sector como a asegurar mejores condiciones y calidad de la educación y el trabajo del personal.

- La definición de marcos e instrumentos de regulación de los mercados laborales, la profesionalización, el ejercicio profesional y los procesos educacionales para el desarrollo del personal.
- El cambio sustantivo de la gestión del personal que permita mejorar la calidad y la productividad del desempeño, con especial énfasis en las redes y servicios descentralizados.

- La garantía en los procesos de formación profesional de la pertinencia social (en términos de valores, actitudes, cultura) y técnica (es decir, en relación con las competencias esenciales y específicas) relacionadas con los modelos de atención vigentes. Ello supone no solo desarrollar las instituciones de educación, sino también asegurar sistemas de educación permanente para el personal de salud en activo.

En síntesis, la nueva generación de reformas deberá incluir objetivos nuevos y darles la prioridad que merecen. Sin perjuicio de las denominaciones concretas que en cada caso adopten, cabe prever la presencia de ocho ejes fundamentales en las futuras agendas de cambio:

1. Fortalecer la infraestructura, las funciones esenciales y la práctica de la salud pública, así como la evaluación de sus procesos y resultados.

2. Fortalecer la función rectora de las autoridades de salud.

3. Ampliar la protección social en el campo de la salud y lograr el acceso universal a los servicios, independientemente de la capacidad individual de pago de los mismos.

4. Propiciar la reorientación de los sistemas y servicios de salud con criterios de promoción de la salud y de prevención de la enfermedad que impriman a los modelos de atención las modificaciones necesarias para que presten servicios con mayor integralidad y mayor énfasis en esos dos aspectos.

5. Mejorar la calidad de la atención.

6. Formar recursos humanos para sustentar los cambios mencionados.

7. Asegurar mecanismos de financiamiento y de asignación de recursos que sean eficientes y solidarios.

8. Impulsar la participación y el control social en los diferentes niveles del sistema de salud.

El desafío consiste en poner en práctica dichas estrategias y lograr un amplio consenso entre todos los actores sobre la importancia de su inclusión en la próxima generación de reformas del sector de la salud. Ello implica redefinir de manera equitativa y solidaria la forma como nuestras sociedades responden institucionalmente a las diferentes necesidades de salud de las personas y de las comunidades, con criterios centrados en la salud de la población y en el bienestar de las personas, más que en el mercado y la eficiencia económica. 